\title{
Parameter Estimation of Human Nerve C-Fibers Using Matched Filtering and Multiple Hypothesis Tracking
}

\author{
Björn Hammarberg (Hansson)*, Student Member, IEEE, Clemens Forster, and Erik Torebjörk
}

\begin{abstract}
We describe how multiple-target tracking may be used to estimate conduction velocity changes and recovery constants of human nerve C-fibers. These parameters discriminate different types of $\mathrm{C}$-fibers and pursuing this may promote new insights into differential properties of nerve fiber membranes.

Action potentials (APs) were recorded from C-fibers in the peroneal nerve of awake human subjects. The APs were detected by a matched filter constituting a maximum-likelihood constant falsealarm rate detector.

Using the multiple-hypothesis tracking method and Kalman filtering, the detected APs (targets) in each trace (scan) were associated to individual nerve fibers (tracks) by their typical conduction latencies in response to electrical stimulation. The measurements were one-dimensional (range only) and the APs were spaced in time with intersecting trajectories. In general, the AP amplitude of each C-fiber differed for different fibers. Amplitude estimation was therefore incorporated into the tracking algorithm to improve the performance.

The target trajectory was modeled as an exponential decay with three unknowns. These parameters were estimated iteratively by applying the simplex method on the parameters that enter nonlinearly and the least squares method on the parameters that enter linearly.
\end{abstract}

Index Terms-Detection, matched filtering, microneurography, multiple-hypothesis tracking, parameter estimation, spike sorting, target tracking.

\section{INTRODUCTION}

I MPROVING signal processing in an application where traditional tools are inadequate often generates many new challenges. We will here describe such an application, arising from the need to examine the stimulus-response characteristics of peripheral unmyelinated (C) fibers in human skin nerves. Key tools for the solution were to be found in a seemingly unrelated area, namely, radar tracking of multiple targets.

Manuscript received April 4, 2001; revised October 23, 2001. This work was supported in part by the Swedish Medical Research Council under Project 5206, in part by the Deutsche Forschungsgemeinschaft under Grant SFB 353. The work of E. Torebjörk was supported by a Max Planck Research Award. Parts of this paper were presented at the Proceedings of the SPIE Conference (AeroSense '98), Signal and Data Processing of Small Targets, 14-16 April 1998 in Orlando, Florida. Asterisk indicates corresponding author.

*B. Hammarberg (Hansson) is with Signals and Systems, Uppsala University, P.O. Box 528, SE-751 20 Uppsala, Sweden, and also with the Department of Clinical Neurophysiology, University Hospital, SE-751 20 Uppsala, Sweden. (e-mail: Bjorn.Hammarberg@ signal.uu.se).

C. Forster is with the Institute of Physiology and Experimental Pathophysiology, University of Erlangen, 91054 Erlangen, Germany.

E. Torebjörk is with the Department of Clinical Neurophysiology, University Hospital, SE-751 20 Uppsala, Sweden.

Publisher Item Identifier S 0018-9294(02)03002-1.

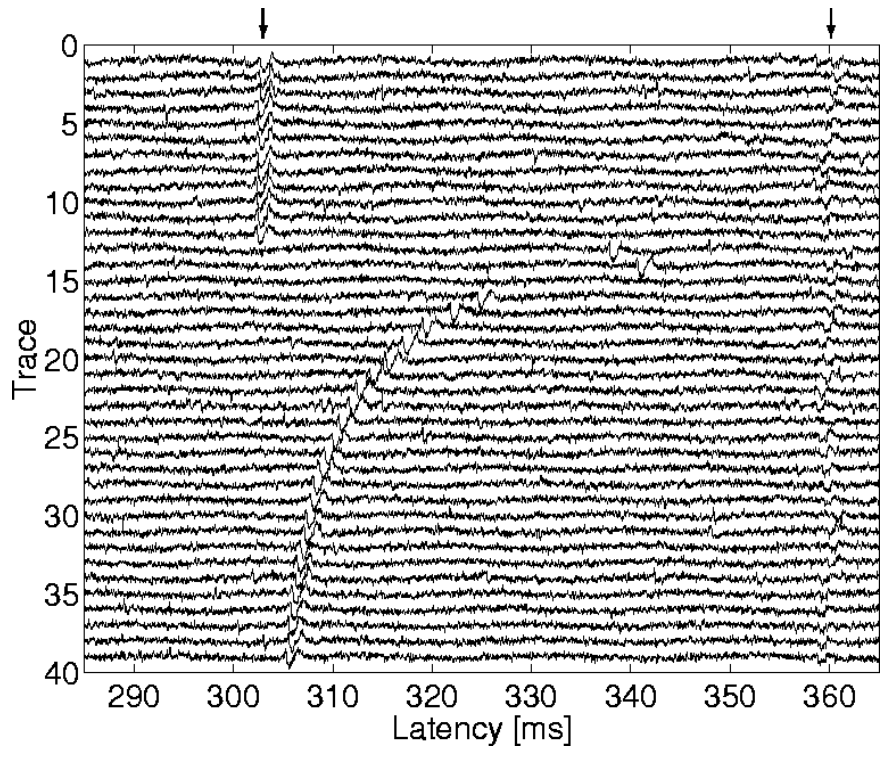

Fig. 1. APs of two C-fibers with latencies of about 303 and $360 \mathrm{~ms}$, respectively. The responses were excited by electrical stimuli delivered at 0.25 $\mathrm{Hz}$ into the skin innervation territory of the fibers. Successive responses are displayed in traces from top to bottom. At trace 13, the left unit was activated in response to a mechanical stimulus causing a decreased conduction velocity, i.e., increased latency. Following this, the conduction velocity recovered gradually as indicated by the APs returning to the latency prior to the activation. The right unit did not respond to the mechanical stimulus and, hence, its latency was retained throughout the recording.

To study the C-fibers, action potentials (APs) are recorded through a thin needle electrode inserted transcutaneously into the peroneal nerve of an awake human subject [1], [2]. Neuronal activity is evoked by applying sensory stimuli in the skin area innervated by the fiber of interest. One problem, however, is that APs originating from other fibers are also recorded by the electrode. Their presence obstructs the examination of the stimulus-response characteristics as it is virtually impossible to decide which APs originate from the fiber under study.

To overcome this problem, Torebjörk and Hallin [3] introduced a method that reveals the excitation of a C-fiber by utilizing the so-called marking phenomenon. The phenomenon stems from the slight decrease of a fiber's conduction velocity after an AP has been conducted. The conduction velocity then slowly returns to its initial value.

The principle of the method is to apply an electrical impulse repetitively, at a low frequency $(0.25 \mathrm{~Hz})$, into the innervation territory of the C-fiber under study. For each impulse, one single AP is evoked and appears in the recording after a certain latency (Fig. 1, at $303 \mathrm{~ms}$ ). To document the response characteristics of 


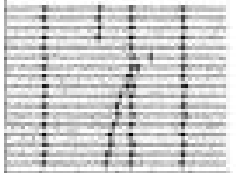

(a)

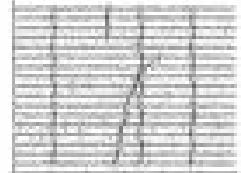

(b)

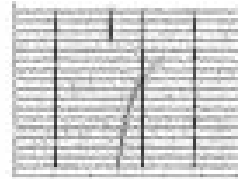

(c)
Fig. 2. Sample results from the three steps of the algorithm. (a) Detected APs. (b) Resulting five tracks after applying the tracking algorithm. (c) Final trajectories obtained by estimating the model parameters.

the C-fiber, a physiological test stimulus (e.g., mechanical, temperature, or chemical) is applied into the receptive field of the fiber. If such a stimulus generates additional APs, the conduction velocity of the affected fiber decreases and the APs excited by the repetitive stimuli show a noticeable increase in latency (Fig. 1, traces 13-40). This change in latency is used as a marker to indicate that the $\mathrm{C}$-unit responded to the applied physiological stimulus [4]. In addition, the latency increase provides information about the number of APs that were generated by the test stimulus [5].

To enhance the efficiency of these experiments, a computersupported recording system is used [6] that both emits the repetitive stimuli and records the responses. Often, several fibers are co-activated and recorded simultaneously, but due to differences in conduction velocity of the individual C-fibers, the APs are spaced in time (Fig. 1, at 303 and $360 \mathrm{~ms}$ ). Using the marking phenomenon, it is thus possible to identify separate $\mathrm{C}$-fibers and to examine their characteristic latency responses.

In the human skin nerves, different types of C-fibers exist [7]. Recently, it has become evident that the latency increase, due to a particular number of impulses, and the time course of their recovery differ in different classes of $\mathrm{C}$-fibers [8]. This finding is intriguing because it may promote new insights into differential properties of membranes in different $\mathrm{C}$-fiber classes in humans.

Previously, the analysis of the recorded traces was carried out manually; a very time consuming task. Therefore, a computer program that detects the APs, discriminates between APs originating from different C-fibers, and estimates latency shifts and recovery constants quantitatively has been developed [9].

In this paper, we present the signal processing algorithms used in the analysis program with emphasis on the AP discrimination. A summary has been presented in [10] and a complete technical report regarding this work is available [11].

\section{AlgORITHM OVERVIEW}

Practical applicability was the most important issue considered during the development of the signal processing algorithms. Hence, optimality was not strived for per se as it often means poor performance if key assumptions become invalid. Instead, the goal was to create an analysis tool that is easy to operate and quickly yields results comparable with a manual analysis. This way, valuable time would be saved for the benefit of the evaluation work.

The signal processing approach we decided upon analyzes the C-fiber recordings in three steps (see Fig. 2).

1) Detection: Signal detection in noise is a problem with well-known solutions. With the considerations mentioned above in mind, the matched filter (MF) was the detector of choice because it is both simple and robust.

2) Tracking: The discrimination step is crucial for the success of this work and it presented an interesting challenge. We found that a reliable algorithm can be derived by exploiting the marking phenomenon and tracking the APs of a particular $\mathrm{C}$-fiber in the responses to the repetitive electrical stimulus; hence, regarding the discrimination problem as a target tracking problem. Due to the vital importance of this step, the multiple-hypothesis tracking (MHT) method [12] was selected because it is commonly considered as the best tracking algorithm.

3) Parameter Estimation: Once the time course of the latency corresponding to a particular C-fiber unit is isolated, a parametric model may be fitted to the data. The current application uses a combination of the Nelder-Mead simplex algorithm and the least squares (LS) method.

This paper is organized as a description of the three main steps of the algorithm. Section III describes how the detection is performed. Section IV deals with the tracking of the APs using the MHT method and Kalman filtering. Moreover, it presents the latency model and the incorporation of the AP amplitude into the tracking algorithm. Section V presents the parameter estimation briefly. Finally, Section VI illustrates the performance on actual recordings obtained from awake human subjects.

\section{TARGET DETECTION}

Detecting signals hidden in high levels of noise is a delicate task. If the signal is a member of a set of signals with known shapes and if the color of the noise is known, then matched filtering constitutes a standard signal processing technique for optimally enhancing and detecting the signal. With this approach, the actual detection is reduced to a simple threshold test where all peaks above a certain detection threshold, here denoted $\tau$, are reported as detections of the sought signal.

To simplify the derivation of the MF, four key assumptions were made.

1) Constant AP Shape: All APs have the same shape and only the amplitude of the APs differ among the units.

2) White Noise: The noise is uncorrelated.

3) Semi-stationary Noise: The noise variance may change from trace to trace, but is constant in each individual trace.

4) "Sparse" Recordings: The energy of any APs in each trace is negligible compared to the noise energy.

Although these assumptions do not fully apply in this particular application, experience has shown that their influence on the overall performance is small.

The first two assumptions reduces the MF design to estimating the AP shape and the noise variance only. The former was done "once and for all" using earlier recordings where several manually detected APs were aligned and averaged to reduce the influence of the noise and the background activity. The averaged potential was then low-pass filtered to further reduce the disturbances in the frequency region where no AP energy is expected. 
The last two assumptions simplifies the noise variance estimation. Currently, a novel maximum-likelihood (ML) estimator is used where the influence of any recorded hum is reduced by applying a notch filter on the data presented to the estimator.

During analysis, the MF output of each trace is normalized by its noise variance to yield a measure of the square root of the instantaneous signal-to-noise ratio (SNR). ${ }^{1}$ For stationary noise, this measure is directly proportional to the amplitude of the AP. The benefits of this design is threefold: First, the detector has a constant false-alarm rate (CFAR). Second, the selection of the detection threshold is simple and may be set by the operator according to the SNR level of the APs being analyzed. Third, the MF output is a scaled amplitude estimate and can be used to improve the tracking performance, see below.

\section{TARGET TRACKING}

Finding a reliable algorithm to discriminate the detected APs was a challenge because traditional methods were not suitable. A pattern matching technique, for example, would have poor performance due to the diminutive differences of the AP shape in these recordings. Moreover, such a technique would disregard the latency information provided by the exploitation of the marking phenomenon.

A clustering algorithm, on the other hand, would indeed use this information, but the time dynamics of active $\mathrm{C}$-fibers would be difficult, if not impossible, to handle.

An experienced eye, however, easily takes full advantage of the marking phenomenon and exploits the latency information in subsequent traces by forming tracks of APs that belong to each other. We decided to mimic this by solving the association problem as a tracking problem using the MHT method with Kalman filtering, see below.

Although the tracks may be found using the latency only, the AP amplitude is utilized to improve the tracking performance. In general, the AP amplitude is different for APs originating from different $\mathrm{C}$-fiber units because the distances between the recording electrode and the nerve fibers differ. Using the scaled amplitude estimate that the MF provides simplifies the design and has performed well.

\section{A. Tracking Algorithm-MHT}

The performance of the tracking algorithm is of vital importance and, hence, we selected the MHT [13] method because it is recognized as the theoretically best approach to multitarget tracking problems. A good presentation of this algorithm and its implementation is found in [12]. For a short introduction, see the Appendix .

The MHT method is a Bayesian probabilistic approach to the tracking problem. For each trace in the recorded data, the detected APs are collected by the tracking system. At a given time and with a given set of detected APs, there are several plausible ways to combine the APs into tracks. Instead of choosing

${ }^{1}$ This is the SNR in the MF output used in the likelihood test and is almost four times larger than the common measure

$$
\mathrm{SNR}_{p p} \triangleq\left(\frac{\text { minimum AP peak-to-peak amplitude }}{\text { noise root-mean-square value }}\right)^{2} \text {. }
$$

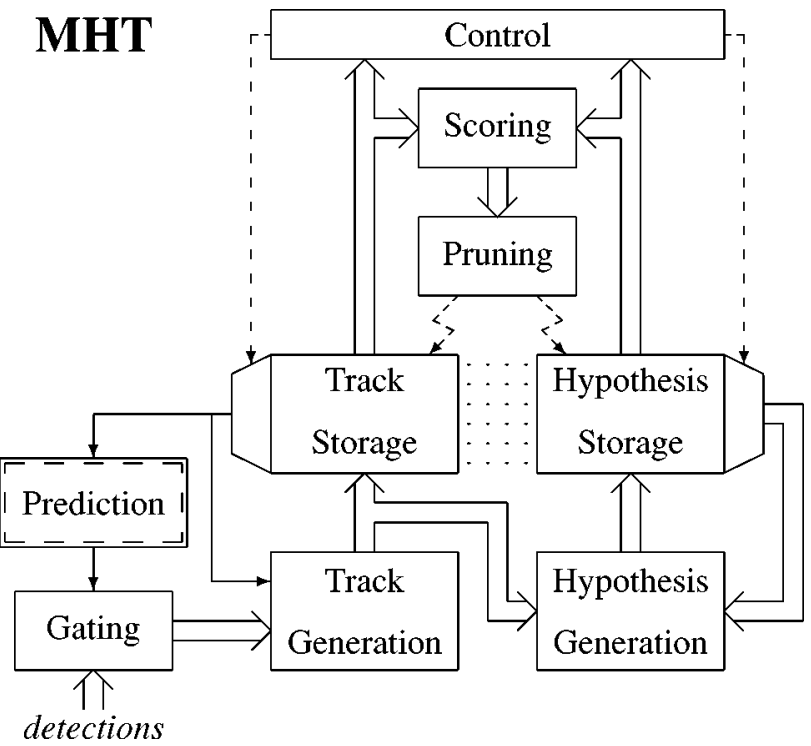

Fig. 3. Block diagram of the MHT algorithm. Data flow is indicated by solid lines, control flow by dashed lines, and other intimate relations by dotted lines. For each trace, the controlling logic iterates over the current track set. The track currently selected is fed to the predictor where the expected AP "position" is calculated. A gate is formed around the prediction and all detections that fall outside the gate are discarded. The current track and the gated detections are used by the track generator to both create updates of the track and to create entirely new tracks. The generated tracks are then stored to be used for the processing of the detections in the next trace. From the hypothesis storage, the control logic selects all hypotheses associated with the current track. They are updated by the hypothesis generator using the generated tracks and are then stored again. When the track iteration is completed, the scores of all hypotheses are calculated and used to prune hypotheses (and tracks) with a low score. The entire process is then repeated for the detections in the next trace. Note that the MHT algorithm does not depend on a particular predictor. The same algorithm may, in principle, be used for any tracking problem. Only the predictor needs to be changed.

only the most probable partitioning after each trace, the MHT method generates a number of candidate hypotheses to be evaluated later when more data are received. Thus, the probability of choosing the correct partitioning of the data into tracks and false alarms is increased.

As Fig. 3 shows, a model of how the AP latencies change from trace to trace is needed to evaluate the probability of each hypothesis. Fig. 1 indicates that the latency is either constant or (approximately) exponentially decreasing. Both cases may be modeled by an exponential model. An expedient way to incorporate this model in the tracking method is presented next.

\section{B. Prediction Algorithm-Kalman Filter}

Fundamental in any tracking system is the track prediction and filtering. The two major alternative methods are the Kalman filter [14] and the filter based on interacting multiple models (IMM) [15]. The IMM method is an extension of the multiplemodel (MM) approach to handle model switching and often provides the best performance. The IMM method is suboptimal, however, and in our application no model switches occur. This suggests that the MM method would give the best performance. At present, the Kalman filter is used, but extending this to an MM-based filtering method is straightforward, if required.

As the process under study is a real physical process assumed to be working in continuous time, a model is based on 
this assumption. To incorporate such a model in the tracking process, a discrete-time model is produced by sampling the continuous-time model [16]. The measurements, however, are modeled as a discrete-time process directly.

1) Process Model: From Fig. 1 it seems reasonable to assume that the measured latency, denoted $y_{1}(t)$, may be modeled by

$$
\begin{aligned}
& y_{1}(t) \triangleq \bar{y}_{1}(t)+\varepsilon(t) \\
& \bar{y}_{1}(t) \triangleq y_{0}+A e^{-\alpha_{0}\left(t-t_{0}\right)}, \quad t \geq t_{0}
\end{aligned}
$$

where $\bar{y}_{1}(t)$ is the true but unknown latency, $y_{0}$ is the latency at steady state, $A$ is the latency shift due to stimulation, $\alpha_{0}$ is the recovery coefficient, $t_{0}$ is the time of excitation, and $\varepsilon(t)$ is the residual consisting of model and measurement errors. By selecting the true latency $\bar{y}_{1}(t)$ and its derivative $\dot{\bar{y}}_{1}(t)$ as state variables, (2) may be written in state space form as an initial value problem with (2) as its solution. This deterministic continuous-time state space model is then augmented with the AP amplitude and replaced by a stochastic continuous-time state space model $^{2}$

$$
\begin{aligned}
\mathbf{d x}_{c}(t) & =\mathbf{A x}_{c}(t) d t+\mathbf{G} \mathbf{d w}(t), \quad t \geq t_{0} \\
\mathbf{A} & \triangleq\left(\begin{array}{ccc}
0 & 1 & 0 \\
0 & -\alpha & 0 \\
0 & 0 & 0
\end{array}\right) \\
\mathbf{G} & \triangleq\left(\begin{array}{ll}
0 & 0 \\
1 & 0 \\
0 & 1
\end{array}\right)
\end{aligned}
$$

where $\mathbf{x}_{c}(t)$ is the three-dimensional continuous-time state vector made up of the latency $\bar{y}_{1}(t)$, its derivative $\dot{\bar{y}}_{1}(t)$, and the MF output $\sqrt{\mathrm{SNR}}$. The 2-D entity $\mathbf{d w}(t)$ is the Wiener increment [17] of a 2-D Wiener process $\mathbf{w}(t)$. The matrix $\mathbf{A}$ is the state transition matrix, in which $\alpha$ should be as close as possible to the true recovery coefficient $\alpha_{0}$.

Because the latency modeling errors are largest at the start of the decay and are almost zero at steady state, the incremental variance of $d w_{1}(t)$ (the first element of $\mathbf{d} \mathbf{w}(t)$ ) is defined as

$$
\begin{aligned}
\sigma_{e_{1}}^{2}(t) d t & \triangleq E\left(d w_{1}(t)\right)^{2} \\
& =\sigma_{e_{1}}^{2}\left(1+e^{\beta_{0}-\beta_{1}\left(t-t_{0}\right)}\right) d t, \quad t \geq t_{0}
\end{aligned}
$$

where $\beta_{i}$ are tuning parameters. This results in a process noise that is high at onset and decreases exponentially to its minimum. Note that this affects the latency part of the system only. For the modeling of the amplitude estimate, a constant incremental variance $\sigma_{e_{2}}^{2} d t$ is used.

Consequently, the incremental covariance matrix $\Lambda(t) d t$ of the Wiener increment $\mathrm{dw}(t)$ is given by

$$
\Lambda(t) d t \triangleq E\left(\mathbf{d} \mathbf{w}(t) \mathbf{d} \mathbf{w}_{(t)}^{T}\right)=\left(\begin{array}{cc}
\sigma_{e_{1}}^{2}(t) & 0 \\
0 & \sigma_{e_{2}}^{2}
\end{array}\right) d t .
$$

${ }^{2}$ The stochastic differential equation (3) may be interpreted as $\dot{\mathbf{x}}_{c}(t)=\mathbf{A} \mathbf{x}_{c}(t)+\mathbf{G e}(t)$, where $\mathbf{e}(t)$ is two-dimensional (2-D) continuous-time white noise.
2) Measurement Model: The measurement model is simply defined as

$$
\mathbf{y}(k)=\mathbf{H x}(k)+\mathbf{v}_{2}(k), \quad \mathbf{H} \triangleq\left(\begin{array}{ccc}
1 & 0 & 0 \\
0 & 0 & 1
\end{array}\right)
$$

where $\mathbf{y}(k)$ is a 2-D measurement vector containing the measured latency and the MF output. The 2-D vector $\mathbf{v}_{2}(k)$ is the measurement noise, modeled as independent zero-mean whitenoise Gaussian processes with a known constant $2 \mid 2$ covariance matrix

$$
\mathbf{Q}_{2} \triangleq E\left(\mathbf{v}_{2}(k) \mathbf{v}_{2}^{T}(k)\right)=\left(\begin{array}{cc}
q_{11}^{(2)} & 0 \\
0 & q_{22}^{(2)}
\end{array}\right) .
$$

3) Initiation and Consistency: Initial estimates of the state and its covariance matrix need to be found before applying the Kalman algorithm. In [11], an LS estimate using two measurements is presented.

Moreover, the model parameters need to be tuned to yield consistent estimates. It is advisable to check this using the methods described in [18].

\section{PARAMETER ESTIMATION}

Estimating model parameters of a particular model once the data is available is straight-forward, in principle. However, the estimation is obstructed somewhat due to the nonlinear data model used here. In the model (2) the parameter $\alpha_{0}$ enters nonlinearly, whereas the two parameters $y_{0}$ and $A$ enter linearly. Our approach is an iterative method in which the nonlinear term is estimated using the simplex method. For each step in the simplex algorithm, the two linear terms are estimated through the LS method and the error norm is returned.

\section{RESULTS}

As stated above, the recordings are processed in three steps by detecting the APs in the recorded traces, tracking their latency changes, and estimating their latency parameters. This section presents the performance of these steps when applied to real recordings. The detection and parameter estimation sections are very brief because they are common methods with well-known characteristics.

\section{A. Matched Filtering}

Despite the erroneous assumptions when deriving the MF, the expected reduction in detection performance has not been a problem in practice. The MF detector is robust in terms of the AP shape and there is no need for retuning the impulse response for different recordings. In addition, it has been incorporated in the on-line data acquisition system to facilitate the experiments.

\section{B. $M H T$}

The most critical part of the overall system is the tracking of the different C-units. For this application to be useful, the number of tracking errors has to be small. Some errors are allowed because erroneous assignments may be corrected by the operator prior to the statistical analysis. Below, two examples are presented: one simple and one more complex. 


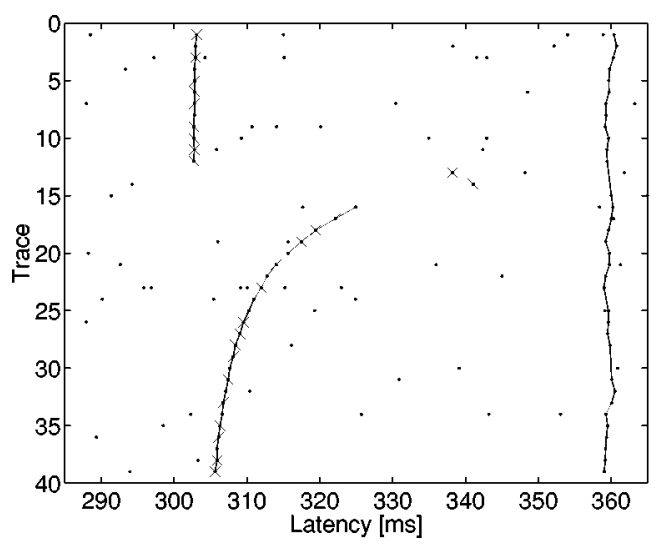

(a)

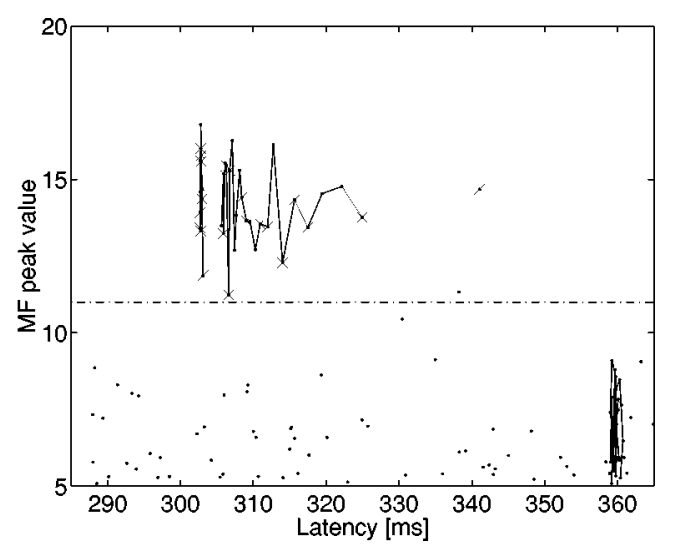

(b)

Fig. 4. Confirmed tracks of the tracker when applied to the two C-fiber unit recording in Fig. 1 ( $\mathrm{SNR}_{p p} \approx 6.8=8.3 \mathrm{~dB}$ ). (a) Time course of the latencies of the units. (b) Amplitude information of the detected APs where the difference between the two units is clearly seen. The APs with an MF peak output above 11 [dashed-dotted in (b)] are marked $(x)$. For comments on the two marked APs in traces 13 and 14, see the text.



(a)

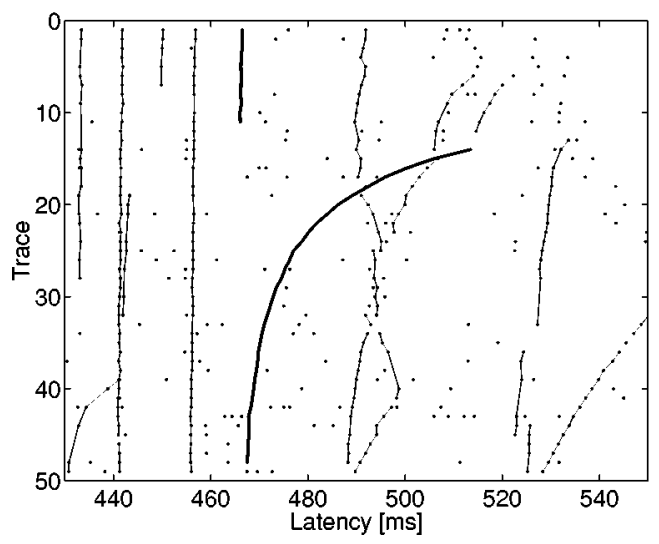

(c)

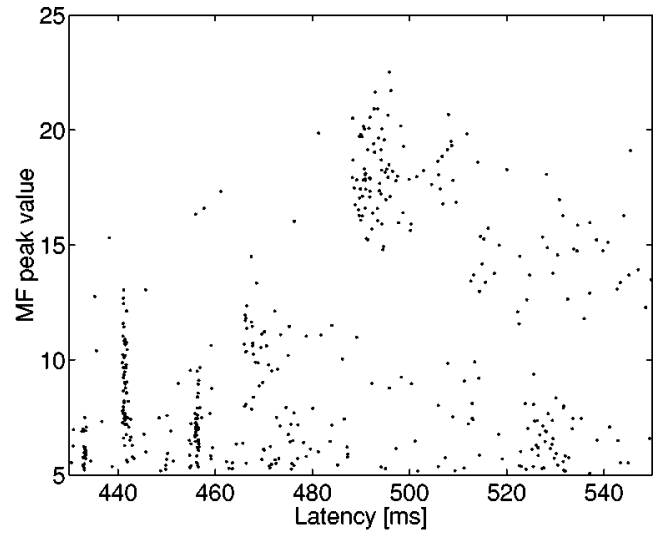

(b)

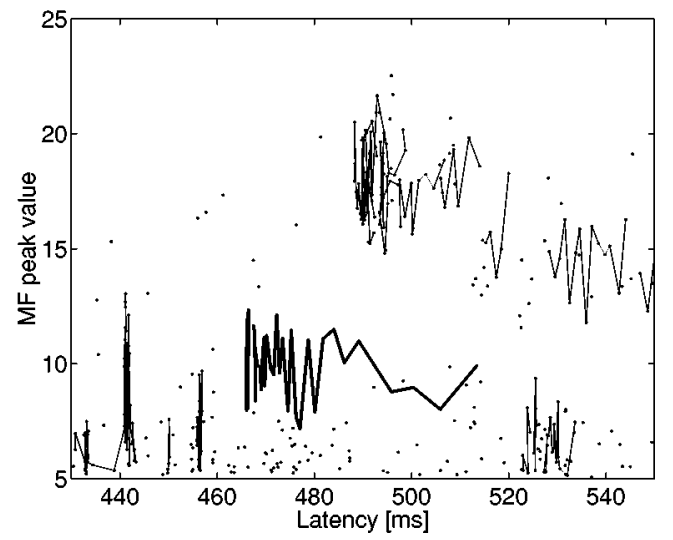

(d)

Fig. 5. Result of the current algorithm where several units were recorded with crossing trajectories ( $\mathrm{SNR}_{p p} \approx 4.0=6.0 \mathrm{~dB}$ ). (a) Time course and (b) amplitude information of the detected APs. (c) and (d) Tracking result is included and the activated unit is marked with a thick line. The method handled crossing tracks well because the amplitudes of the APs differed.

1) A Two Unit Recording: This basic example involves two well-separated units: one with high amplitude and one with low amplitude, see Fig. 1. After matched filtering, we obtain the data shown in Fig. 4 where each dot represents a MF output above the selected detection threshold $\tau=5$. First, both units were inactive and their latencies were constant at about 303 and $360 \mathrm{~ms}$, respectively. At trace 13, the high-amplitude unit was activated by a mechanical stimulus and its latency increased dramatically.
The latency then slowly recovered to the level before the activation.

As shown in Fig. 4, the algorithm successfully tracked both units. Note that the track of the low-amplitude unit was not lost despite that APs were missed in some traces.

Note also the two high-amplitude AP detections in traces 13 and 14 not assigned to any track. They probably originated from the high-amplitude unit, but because they were found in 
the traces directly after the activation of the unit, it is not clear whether they corresponded to the electrical impulses, the mechanical stimulus, or some spurious after effects of the mechanical stimulus. Hence, this result is considered as "optimal" or at least near "optimal".

If only the high-amplitude unit had been of interest, the tracking would have been simplified by choosing a higher threshold. For example, using $\tau=11$, only the $\times$-marked APs in the diagrams of Fig. 4 would have been detected and processed.

To get the best tracking result possible, the Kalman filter settings used on this recording were tuned to their "optimal" values for this particular unit. By estimating the parameters of the active unit's recovery trajectory and using the consistency analysis described in [18], the Kalman filter was subjectively tuned to give as consistent estimates as possible. The selected parameters were

$$
\begin{aligned}
\alpha & =0.0375\left[\mathrm{~ms}^{-1}\right] \quad \beta_{1}=0.05\left[\mathrm{~ms}^{-1}\right] \\
\sigma_{e_{1}}^{2} & =2 \cdot 10^{-5}\left[\mathrm{~ms}^{-1}\right] \quad q_{11}^{(2)}=0.05\left[\mathrm{~ms}^{2}\right] \\
\sigma_{e_{2}}^{2} & =10^{-7}\left[\mathrm{~ms}^{-1}\right] \quad q_{22}^{(2)}=1 \\
\beta_{0} & =3 .
\end{aligned}
$$

In principle, a robust Kalman filter setting that is optimal for all units is desired. Experience has shown, however, that this is not crucial to the performance (see, for example, below).

2) A Multiunit Recording: This example is more complex and more realistic and involves several C-units with crossing trajectories. Several of the units were inactive during the recording, and one of the units (drawn with a thick line) was strongly activated between trace 11 and trace 12, see Fig. 5 . Moreover, there were two spontaneously active sympathetic $\mathrm{C}$-units that may be recognized by their more irregular behavior.

It is evident from Fig. 5 that a good tracking result was obtained for the activated unit as well as for the inactive ones. In this example, no parameter estimation was done to tune the algorithms. Instead, the algorithm parameters were identical to the ones used in the previous example.

Moreover, the importance of the amplitude information should be obvious as the latency trajectory of the active unit crossed the two sympathetic units, but their amplitudes differed.

Note also the tracker's ability to discriminate between the two tracks at about $440 \mathrm{~ms}$ (traces 20-30) despite their closeness and low SNR levels.

Note, finally, the lost track between traces 45 and 46 (latency $440 \mathrm{~ms}$ ). This tracking error may, however, be corrected by the operator prior to the parameter estimation.

From the perspective of the researchers carrying out these experiments, this is a really good result. The otherwise manually task of discriminating the APs is here quickly performed automatically and reliably.

\section{Parameter Estimation}

Fitting an exponential curve to the time course of the latency is straight-forward. The LS-simplex method chosen here performs well and yields good parameter estimates.

\section{DISCUSSION}

An application of matched filtering and the MHT method to estimate parameters of human nerve C-fiber APs was presented. The objective of the algorithm was to automate the classification of recorded APs in order to quantitatively estimate conduction latency shifts and post-stimulus recovery constants of the C-fiber units.

The APs were successfully detected by means of an MF constituting a ML-CFAR detector. Even APs with amplitudes of the same order of magnitude as the peaks of the noise were detected correctly with a reasonably low false-alarm rate.

The discrimination between APs originating from different C-fiber units was carried out using the MHT method described in [12]. Only some minor changes were introduced to adapt the method to the application described. The results corresponded well with what an experienced physician considers to be a "correct" result. Two years experience also show that the classification is in general correct and that the need for operator intervention is limited.

The estimation of the model parameters was straight-forward using an exponential decay model. The combination of the simplex method and the LS method worked well even for short recovery segments.

In spite of the good performance, there are some aspects that could be improved. In the MF derivation, for example, the noise was assumed to be white. As this is not the case in real recordings, the color of the noise should have been considered when the MF impulse response was tuned. Despite this deficiency, the MF detector has shown to perform well on the real recordings as well as to be robust to a wide range of AP shapes.

The most obvious drawback of the MHT algorithm is its exhaustive computational and storage requirements. In the cases considered so far, these requirements have, however, not been a limiting factor. If they were, the track oriented MHT implementation could be used as its computational and storage requirements are less demanding.

The reason for choosing the MHT method in spite of its complexity is that it is considered to be one of the best tracking methods regarding to performance. Other methods have various shortcomings. For example, the low track maintenance of the nearest neighbor association method, and the typical track switching behavior of the Joint Probabilistic Data Association method are not acceptable, see [19].

Another drawback of the current implementation is its sensitivity to errors in the model parameter $\alpha$ that represents an a priori value of the recovery time constant of the latency. It is reasonable to believe, however, that introducing robust filtering methods or replacing the Kalman predictor with an MM-based predictor would make the filtering less sensitive to differences in the recovery constant. Implementing the MM approach is probably the most straight-forward because the number of observations to initiate the predictor is kept low. Otherwise, some iterative initiation procedure would have to be included.

The parameter estimation residuals, finally, were neither Gaussian nor white due to model errors. Moreover, the estimated confidence intervals tended to be larger than the true confidence intervals. These deficiencies existed because the 
parameter estimation is based on a theory that is only asymptotically correct and because the trajectory lengths considered in this type of application are too short in this regard.

To further improve the performance of the algorithm, future work should include a noise covariance matrix estimator and an MM-based target predictor. If possible, a better model of the latency recovery together with a more appropriate statistical analysis of the parameter estimation would be valuable.

Nonetheless, the goal of simplifying and improving the efficiency of the analysis of the human nerve $\mathrm{C}$-fiber recordings was reached. The computer application developed based on the algorithms presented in this paper has already shown to be useful to the research team. In the practical cases considered so far, the overall performance of the application has been very good.

\section{APPENDIX}

In this appendix, a short introduction to the MHT method is given.

As signal processing in general, the results of a target tracking method depend on the amount of data available; the more data or observations available, the better the tracking result.

The main idea of the MHT method is to achieve high performance by considering all observations before deciding upon a particular partitioning of the collected data. This is done by enumerating all possible ways of combining the observations into tracks and false alarms, and then choosing the partitioning that is best. By noting that each combination forms a hypothesis, the a posteriori probability may be calculated and used to rank the hypothesized partitionings.

The obvious drawback of this method is its exhaustive computational and storage requirements. It is, however, possible to alleviate the demands and [12] describes three approaches: gating, clustering, and pruning. The first approach, gating, aims at limiting the number of hypotheses by avoiding combinations of observations that are very distant from each other because such observations are very unlikely to originate from the same target.

The second approach, clustering, aims at limiting the number of hypotheses by separating noninteracting hypotheses into independent clusters. Instead of solving one large tracking problem, a number of smaller problems are then solved separately.

The third and last approach, pruning, aims at bringing down the exponential rate of increase in number of hypotheses by discarding unlikely hypotheses as soon as possible. This decreases the number of hypotheses that give rise to new ones.

To be able to calculate the probabilities of the hypotheses, one has to know some statistics about the data to partition. Moreover, a model is needed that describes how the observations in a particular track change from trace to trace.

\section{ACKNOWLEDGMENT}

The authors would like to thank Prof. M. Sternad and Prof. A. Ahlén for valuable comments regarding this work.

\section{REFERENCES}

[1] H. E. Torebjörk and R. G. Hallin, "C-fiber units recorded from human sensory nerve fascicles in situ," Acta Soc. Med. Ups., vol. 75, pp. 81-84, 1970.

[2] R. G. Hallin and H. E. Torebjörk, "Afferent and efferent C units recorded from human skin nerves in situ," Acta Soc. Med. Ups., vol. 75, pp. 277-281, 1970.

[3] H. E. Torebjörk and R. G. Hallin, "Responses in human A and C fibres to repeated electrical intradermal stimulation," J. Neurol. Neurosurg. Psy., vol. 37, pp. 653-664, 1974.

[4] M. Schmelz, R. Schmidt, M. Ringkamp, C. Forster, H. O. Handwerker, and H. E. Torebjörk, "Limitation of sensitization to injured parts of receptive fields in human skin C-nociceptors," Exp. Brain Res., vol. 109, pp. 141-147, 1996.

[5] M. Schmelz, C. Forster, R. Schmidt, M. Ringkamp, H. O. Handwerker, and H. E. Torebjörk, "Delayed responses to electrical stimuli reflect C-fiber responsiveness in human microneurography," Exp. Brain Res., vol. 104, pp. 331-336, 1995.

[6] C. Forster and H. O. Handwerker, "Automatic classification and analysis of microneurographic spike data using a PC/AT," J. Neurosci. Meth., vol. 31, pp. 109-118, 1990.

[7] R. Schmidt, M. Schmelz, C. Forster, M. Ringkamp, H. E. Torebjörk, and H. O. Handwerker, "Novel classes of responsive and unresponsive C nociceptors in human skin," J. Neurosci., vol. 15, pp. 333-341, 1995.

[8] C. Weidner, M. Schmelz, B. Hansson, H. O. Handwerker, and H. E. Torebjörk, "Functional attributes discriminating mechano-insensitive and mechano-responsive C nociceptors in human skin," J. Neurosci., vol. 19, pp. $10184-10190$, Nov. 1999.

[9] K. Lundin, "A System for Analysis of Human Pain Signals using a Radar Tracking Approach," masters thesis, Uppsala Univ., Uppsala, Sweden, 1998.

[10] B. Hansson, C. Forster, and E. Torebjörk, "Matched filtering and multiple hypothesis tracking applied to C-fiber action potentials recorded in human nerves," in Proc. SPIE, Signal and Data Processing of Small Targets, vol. 3373, Orlando, FL, Sept. 1998, pp. 582-593.

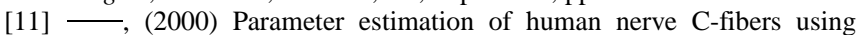
matched filtering and multiple hypothesis tracking: Complete report, Uppsala, Sweden. [Online]. Available: http://www.signal.uu.se/Publications/abstracts/r005.html

[12] S. S. Blackman, Multiple-Target Tracking with Radar Applications. Dedham, MA: Artech House, 1986.

[13] D. B. Reid, "An algorithm for tracking multiple targets," IEEE Trans. Automat. Contr., vol. AC-24, pp. 843-854, Dec. 1979.

[14] S. Haykin, Adaptive Filter Theory, 2nd ed. Englewood Cliffs, NJ: Prentice-Hall, 1991.

[15] D. Lerro and Y. Bar-Shalom, "Interacting multiple model tracking with target amplitude feature," IEEE Trans. Aerosp. Electron. Syst., vol. 29, pp. 494-508, Apr. 1993.

[16] K. J. Åström and B. Wittenmark, "Discrete-time systems," in Computer Controlled Systems, Theory and Design, 3rd ed. Upper Saddle River, NJ: Prentice-Hall, 1997, pp. 30-76.

[17] K. J. Åström, "Stochastic state models," in Introduction to Stochastic Control Theory. New York: Academic, 1970, pp. 44-90.

[18] Y. Bar-Shalom and X.-R. Li, Estimation and Tracking: Principles, Techniques, and Software. Norwood, MA: Artech House, 1993.

[19] M. de Feo, A. Graziano, R. Miglioli, and A. Farina, "IMMJPDA versus MHT and Kalman filter with NN correlation: Performance comparison," Inst. Elect. Eng. Proc, Radar, Sonar Navigat., vol. 144, no. 2, pp. 49-56, 1997.

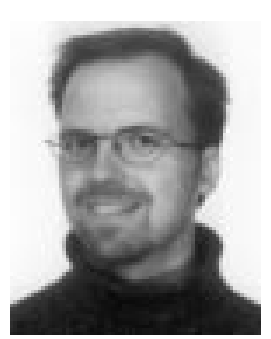

Björn Hammarberg (Hansson) ( $\left.\mathrm{S}^{\prime} 00\right)$ was born in Östersund, Jämtland, Sweden, in 1970. He received the M.Sc. degree in engineering physics from Uppsala University, Uppsala, Sweden, in 1995. Since 1996, he has been working towards the Ph.D. degree in signal processing of neurophysiological data at the Signals and Systems, Uppsala University.

Continuously since 1995, he has also been working at the Department of Clinical Neurophysiology at University Hospital, Uppsala, with signal processing in various medical applications. During the fall of 1995, he was at the Department of Clinical Neurophysiology, Institute of Neurology, Nijmegen University Hospital, Nijmegen, The Netherlands, working on an EMG decomposition system. 


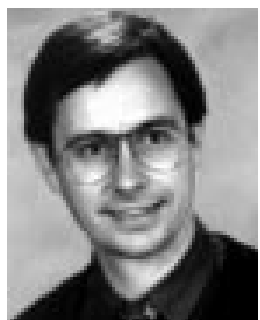

Clemens Forster received the M.S. degree in computational science in medicine and the Ph.D. degree in biomedical science, from the University of Heidelberg, Heidelberg, Germany, in 1984 and 1991, respectively.

He worked for several years as a Research Assistant in the development of systems for recording and analysis of neurophysiological data. He is currently with the Department of Physiology and Experimental Pathophysiology, the University of Erlangen-Nuernberg, Erlangen, Germany, where he is a Member of the Pain Research Group. His main research interests are in the fields of biomedical signal processing and analysis of pain-related brain areas using functional magnetic resonance imaging.

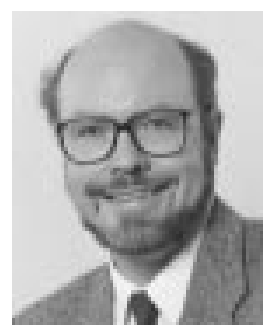

Erik Torebjörk is Professor of Pain Research at the University of Uppsala, Uppsala, Sweden, and Consultant of Clinical Neurophysiology at the University Hospital in Uppsala.

$\mathrm{He}$ has published extensively on neurophysiological pain mechanisms in humans with particular interest focused on the function of unmyelinated nociceptive C-fibers. 